\title{
A chiral quark-soliton model with broken scale invariance for nuclear matter
}

\author{
V. Mantovani Sarti and A. Drago
}

Dipartimento di Fisica, Università di Ferrara and INFN, Sezione di Ferrara via Saragat 1, 44121 Ferrara, Italy

ricevuto il 31 Agosto 2012

\begin{abstract}
Summary. - Soliton models based on the linear $\sigma$-model fail to describe nuclear matter already at $\rho \sim \rho_{0}$ due to the restrictions on the scalar field dynamics imposed by the Mexican hat potential. To overcome this problem we used a chiral Lagrangian, including a logarithmic potential associated with the breaking of scale invariance, based on quarks interacting with chiral fields, $\sigma$ and $\pi$, and with vector mesons. Using the Wigner-Seitz approximation to mimic a dense system, we show that the model admits stable solitonic solutions at higher densities with respect to the linear- $\sigma$ model and that the introduction of vector mesons allows to obtain saturation. This result has never been obtained before in similar approaches.
\end{abstract}

PACS 12.39.Fe - Chiral Lagrangians.

PACS 21.65.Mn - Nuclear matter-equations of state.

PACS 21.30.Fe - Hadrons-nuclear forces.

\section{1. - The model}

We consider the following Lagrangian $[1,2]$ :

$$
\begin{aligned}
\mathcal{L}_{V M}= & \bar{\psi}\left(i \gamma^{\mu} \partial_{\mu}-g\left(\sigma+i \boldsymbol{\pi} \cdot \boldsymbol{\tau} \gamma_{5}\right)+g_{\rho} \gamma^{\mu} \frac{\boldsymbol{\tau}}{2} \cdot\left(\boldsymbol{\rho}_{\mu}+\gamma_{5} \boldsymbol{A}_{\mu}\right)-\frac{g_{\omega}}{3} \gamma^{\mu} \omega_{\mu}\right) \psi \\
& +\frac{\beta}{2}\left(D_{\mu} \sigma D^{\mu} \sigma+D_{\mu} \boldsymbol{\pi} \cdot D^{\mu} \boldsymbol{\pi}\right)-\frac{1}{4}\left(\boldsymbol{\rho}_{\mu \nu} \cdot \boldsymbol{\rho}^{\mu \nu}+\boldsymbol{A}_{\mu \nu} \cdot \boldsymbol{A}^{\mu \nu}+\omega_{\mu \nu} \omega^{\mu \nu}\right) \\
& +\frac{1}{2} m_{\rho}^{2}\left(\boldsymbol{\rho}_{\mu} \cdot \boldsymbol{\rho}^{\mu}+\boldsymbol{A}_{\mu} \cdot \boldsymbol{A}^{\mu}\right)+\frac{1}{2} m_{\omega}^{2} \omega_{\mu} \omega^{\mu}-V\left(\phi_{0}, \sigma, \pi\right)
\end{aligned}
$$

Here $\psi$ is the quark field, $\sigma$ and $\pi$ are the chiral fields, $\omega_{\mu}$ is a vector-isoscalar coupled to baryon current, $\boldsymbol{\rho}_{\mu}$ and $\boldsymbol{A}_{\mu}$ are respectively a vector-isovector and an axial-vectorisovector fields coupled to isospin and axial-vector current. Here $\phi$ is the dilaton field 

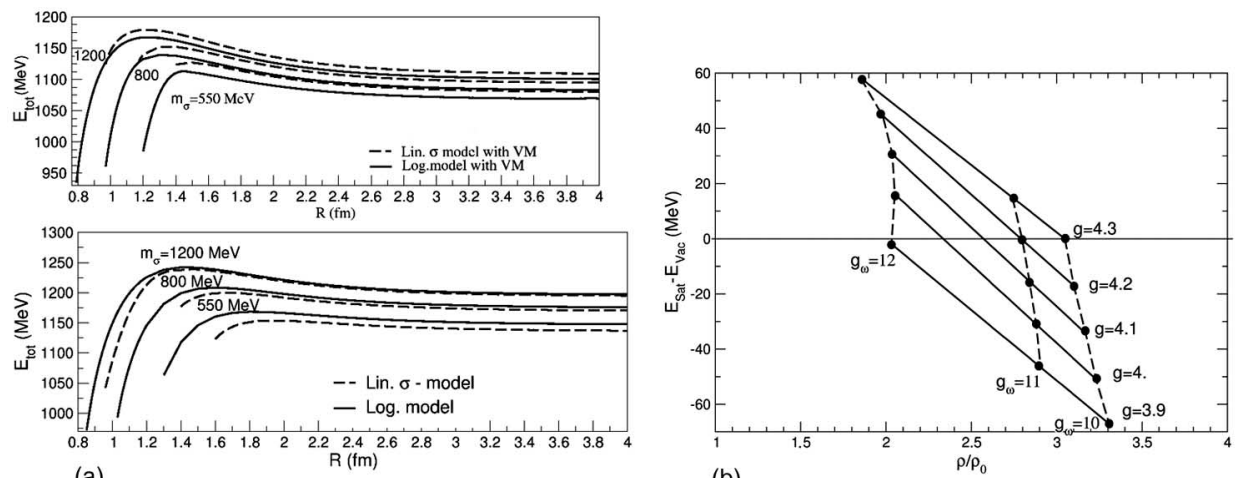

(b)

Fig. 1. - (a) Total energy of the soliton as a function of the cell radius $R$ in the model without (upper panel) and with vector mesons (lower panel). (b) Minimum of the total energy as a function of the density.

which, in the present calculation, is kept frozen at its vacuum value $\phi_{0}[1,2]$. The logarithmic potential reads

$$
\begin{aligned}
V(\sigma, \pi) & =\lambda_{1}^{2}\left(\sigma^{2}+\pi^{2}\right)-\lambda_{2}^{2} \ln \left(\sigma^{2}+\pi^{2}\right)-\sigma_{0} m_{\pi}^{2} \sigma \\
\lambda_{1}^{2} & =\frac{1}{4}\left(m_{\sigma}^{2}+m_{\pi}^{2}\right), \lambda_{2}^{2}=\frac{\sigma_{0}^{2}}{4}\left(m_{\sigma}^{2}-m_{\pi}^{2}\right) .
\end{aligned}
$$

The masses of bare fields are: $m_{\pi}=139 \mathrm{MeV}, m_{\rho}=m_{A}=776 \mathrm{MeV}$ and $m_{\omega}=$ $782 \mathrm{MeV}$. For the sigma field, since there are no experimental constraints, we use $m_{\sigma}=$ $550 \mathrm{MeV}$ and $m_{\sigma}=1200 \mathrm{MeV}$. We fixed $g_{\rho}=4$ and we vary $g_{\omega}$ between 10 and 13 . The pion-quark coupling constant $g$ will vary from 3.9 to 5 . The calculation is performed at Mean-Field level by adopting the hedgehog ansatz for the fields.

\section{2. - The Wigner-Seitz approximation to nuclear matter}

The Wigner-Seitz approximation consists of building a spherical symmetric lattice where each soliton sits on a spherical cell of radius $R$ with specific boundary conditions imposed on fields at the surface of the sphere. In particular here we adopt the choice of ref. [3] which relates the boundary conditions at $R$ to the parity operation, $\boldsymbol{r} \rightarrow-\boldsymbol{r}$. The presence of a periodical lattice implies the formation of a band structure. Here we evaluate the band width in two different approaches following ref. [4].

\section{3. - Results}

3*1. The effect of the dilaton potential: going beyond $\rho_{0}$. - In fig. $1($ a) we show how the total energy of the soliton varies as $R$ decreases:

- for each value of $m_{\sigma}$, the logarithmic model (solid line) admits solitonic solutions for smaller values of the cell radius $R$ (e.g. higher densities) in comparison to the linear- $\sigma$ model (dashed line);

- the introduction of vector mesons stabilises the solutions at high densities. 
3`2. Getting saturation at finite density. - The saturation at finite density is obtained by including also the band effect in the evaluation of the total energy and the scenario we obtain is the following:

- the repulsive effect of vector meson prevails up to $\rho \approx \rho_{0}$ while at higher densities the band effect, connected to the sharing of quarks between solitons, provides the dominant contribution to repulsion (for more details see ref. [5]);

- this mechanism, as shown in fig. 1(b) is stable with respect to a wide range of parameters, $g$ and $g_{\omega}$, and moreover this range partially overlaps the one that provides a reasonable description of the single soliton [6].

\section{REFERENCES}

[1] Heide E. K., Rudaz S. and Ellis P. J., Nucl. Phys. A, 571 (1994) 713; Carter G. W., Ellis P. J. and Rudaz S., Nucl. Phys. A, 603 (1996) 367; Carter G. W., Ellis P. J. and Rudaz S., Nucl. Phys. A, 618 (1997) 317; Carter G. W. and Ellis P. J., Nucl. Phys. A, 628 (1998) 325.

[2] Bonanno L. and Drago A., Phys. Rev. C, 79 (2009) 045801.

[3] Weber U. and McGovern J. A., Phys. Rev. C, 57 (1998) 3376.

[4] Hahn D. and Glendenning N. K., Phys. Rev. C, 36 (1987) 1181; Birse M. C., Rehr J. J. and Wilets L., Phys. Rev. C, 38 (1988) 359.

[5] Drago A. and Sarti V. M., arXiv:1109.5399 [nucl-th].

[6] Broniowski W. and Banerjee M. K., Phys. Rev. D, 34 (1986) 849. 